\title{
Comparison of linear and nonlinear active disturbance rejection control method for hypersonic vehicle
}

\author{
JIA Song ${ }^{1}, \mathrm{KE} \mathrm{Gao}^{1}$, LUN Wang ${ }^{1}$, ERFU Yang ${ }^{2}$ \\ 1. School of Astronautics, Beihang University, Beijing 100191, China \\ E-mail: songjia@buaa.edu.cn \\ 2. Space Mechatronic Systems Technology Laboratory Strathclyde Space Institute, University of Strathclyde Glasgow, UK \\ E-mail: erfu.yang@strath.ac.uk
}

\begin{abstract}
Near space hypersonic vehicles have features of strong coupling, nonlinearity and acute changes in aerodynamic parameters, which are challenging for the controller design. Active disturbance rejection control (ADRC) method does not depend on the accurate system model and has strong robustness against disturbances. This paper discusses the differences between the fractional-order PID (FOPI ${ }^{\lambda}{ }^{\mu}$ ) ADRC method and the FOPI ${ }^{\lambda} \mathrm{D}^{\mu}$ LADRC method for hypersonic vehicles. The $\mathrm{FOPI}^{\lambda} \mathrm{D}^{\mu} \mathrm{ADRC}$ controller in this paper consists of a tracking-differentiator (TD), a FOPI ${ }^{\lambda} \mathrm{D}^{\mu}$ controller and an extended state observer (ESO).The FOPI ${ }^{\lambda} D^{\mu}$ LADRC controller consists of the same TD and FOPI ${ }^{\lambda}{ }^{\mu}$ controller with the FOPI ${ }^{\lambda} D^{\mu}$ ADRC controller and a linear extended state observer (LESO) instead of ESO. The stability of LESO and the FOPI ${ }^{\lambda} \mathrm{D}^{\mu}$ LADRC method is detailed analyzed. Simulation results show that the FOPI ${ }^{\lambda} \mathrm{D}^{\mu}$ ADRC method can make the hypersonic vehicle nonlinear model track desired nominal signals faster and has stronger robustness against external environmental disturbances than the FOPI ${ }^{\mu}{ }^{\mu}$ LADRC method.
\end{abstract}

Key Words: nonlinear active disturbance rejection control, active disturbance rejection control, FOPI $^{\lambda} \mathrm{D}^{\mu}$ control, near space hypersonic vehicle

\section{Introduction}

Near space hypersonic vehicles have potential values in both military and civil applications and have received much attention in recent years [1]. Compared to the traditional aerial vehicles, hypersonic vehicles are characterized by large envelops, high speed, low launch cost, dynamics and reusability [2]. However, their features of nonlinearity, strong coupling and aerodynamic uncertainty may lead to poor robustness properties of the closed-loop control systems, and thereby result in challenging for the robust controller design [3].

Many control methods have been discussed to achieve the flight control of the hypersonic vehicles during the last two decades. In [4], an adaptive output feedback controller was presented and applied to a linearized hypersonic vehicle model, and simulation results showed good tracking performance with the controller. A control method based on aero propulsive and elevator-to-lift couplings was proposed in [5] for an air breathing hypersonic vehicle and simulation results showed good performance of the controller. In [6], a linear parameter-varying theory based on the fractional transformation model was applied to design the controller for a hypersonic reentry vehicle, and simulations showed the accuracy and robustness of the proposed closed-loop control system for hypersonic reentry vehicles. An approximate back-stepping fault-tolerant controller was designed in [7] for a flexible air-breathing hypersonic vehicle and simulation results demonstrated good tracking properties. A composite controller was proposed in [8] for an air-breathing hypersonic vehicle to achieve the velocity and height

*This work is supported by National Science Foundation project (Contract No. 51206007) and Aeronautical Science Foundation of China (2013ZC51). tracking control. Duan and Li [9] summarized the limitations of some control methods on high quality and realization.

The active disturbance rejection control (ADRC) method proposed by Han [10] using the dynamic feedback compensation for the lumped unknown disturbances. Inherited from a proportion-integral-derivative (PID) method, the ADRC method is to address the weaknesses of PID and has some advantages on robustness and anti-disturbance, and has been widely used in many fields. The active disturbance rejection control (ADRC) is now considered as a powerful control strategy in dealing with large uncertainty covering unknown dynamics, external disturbance, and unknown part in coefficient of the control [11]. In [12], a modified ADRC method was used in a 6-degree-of-freedom parallel platform and the platform could be driven to follow the given references well. Furthermore, the ADRC method has been used in magnetic rodless pneumatic cylinder [13], electromagnetic linear actuator [14], multimotor servomechanism [15] and magnetic bearing [16]. However, compared with the traditional ADRC method which is nonlinear, a linear active disturbance rejection control (LADRC) method has also been developed for controller design. The experiment results of $[17,18,19,20,21]$ which used linear ADRC method all have achieved good results. In this paper, we clarify and analysis the structure differences, characteristic differences etc. of the FOPI ${ }^{\mu}{ }^{\mu}$ ADRC method and $\mathrm{FOPI}^{\lambda} \mathrm{D}^{\mu}$ LADRC. However, the ADRC method and the LADRC method both have more tuning parameters than the traditional PID method, while the appropriate controller parameters depend on the experiences of experts. Sometime, the number of the ADRC method parameters can be reduced to one or two $[16,20]$. To compare the traditional ADRC method and the LADRC method clearly, this paper does not reduce parameters. 
The remainder of the paper is organized as follows. In Section 2, the hypersonic vehicle vertical model (VM) is established. In Section 3, the differences between the FOPI ${ }^{\lambda} D^{\mu}$ ADRC method and $\mathrm{FOPI}^{\lambda} \mathrm{D}^{\mu}$ LADRC are analyzed and clarified, the stability of LESO and FOPI ${ }^{\lambda} \mathrm{D}^{\mu}$ LADRC controller is detailed discussed. In section 4, verification simulation analysis results are shown. Finally, Section 5 draws conclusions.

\section{Hypersonic vehicle vertical model}

This paper uses the generic hypersonic vehicle (GHV) as the control object [22]. The aerodynamic equations and model parameters are obtained from [23]. The atmospheric model refers to the U.S. standard atmosphere 1976. The three-view drawing is shown in Fig. 1 and the notations related to GHV are shown in Fig. 2, according to [24].

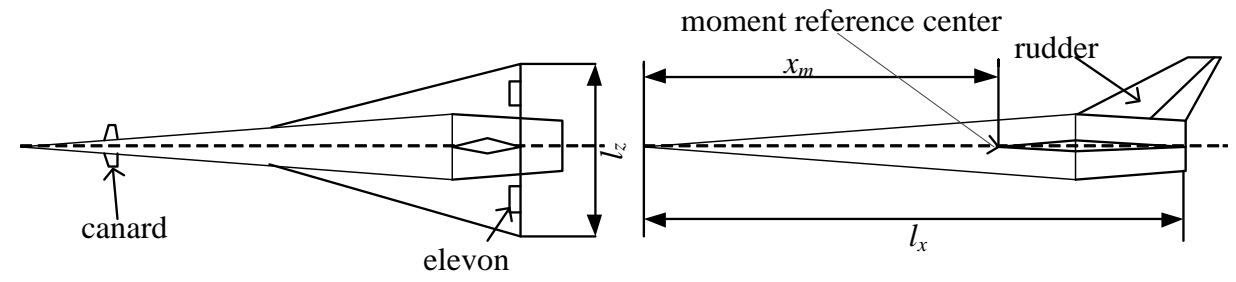

Fig. 1: Three view of the GHV

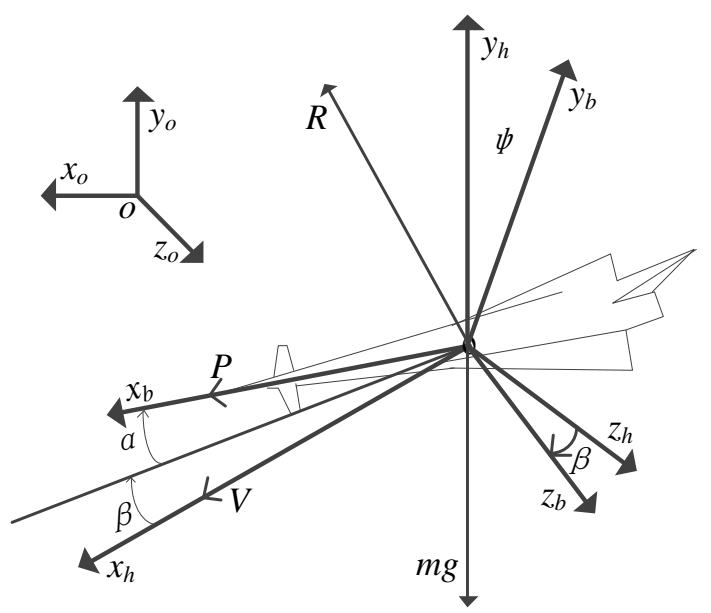

Fig. 2: Notations related to GHV

In Fig. 2, $o x_{o} y_{o} z_{o}, o x_{h} y_{h} z_{h}$ and $o x_{b} y_{b} z_{b}$ denote the inertia coordinate system, the speed coordinate system and the body axes coordinate system, respectively, $m, R$ and $P$ denote the mass of vehicle, aerodynamic force and propulsion, respectively, $\alpha, \beta$ and $V$ represent the attack angle, sideslip angle and velocity, respectively, $\vartheta, \psi$ and $\gamma$ denote the pitch angle, yaw angle and roll angle, respectively.

Therefore, the pitch channel equation can be written as follows:

$$
\left\{\begin{aligned}
\dot{\alpha}= & \omega_{\mathrm{z}}-\omega_{\mathrm{x}} \cdot \cos \alpha \cdot \tan \beta+\omega_{\mathrm{y}} \cdot \sin \alpha \cdot \tan \beta \\
& \quad-(\mathrm{Lsec} \beta+\mathrm{mg} \cos \theta) / \mathrm{mV} \\
\dot{\omega}_{\mathrm{z}}= & \left(\mathrm{I}_{\mathrm{x}}-\mathrm{I}_{\mathrm{y}}\right) / \mathrm{I}_{\mathrm{z}} \cdot \omega_{\mathrm{x}} \cdot \omega_{\mathrm{z}}+\mathrm{N} / \mathrm{I}_{\mathrm{z}} \\
\vartheta= & \theta+\alpha
\end{aligned}\right.
$$

Where $\omega_{x}, \omega_{y}$ and $\omega_{z}$ represent roll, yaw and pitch angular rate, respectively, $L$ and $N$ represent lift force and pitch moment, respectively, $I_{x}, I_{y}$ and $I_{z}$ represent $x, y$ and $z$ coordinate moment of inertia, respectively. In this paper, fuel slosh is not considered and the products of inertia are neglected in order to simplify the vehicle model.

\section{Comparation of $\mathrm{FOPI}^{\lambda} \mathrm{D}^{\mu}$ ADRC method and FOPI ${ }^{\lambda} D^{\mu}$ LADRC method}

\subsection{FOPI $^{\lambda} D^{\mu} \mathrm{ADRC}$ and $\mathrm{FOPI}{ }^{\lambda} \mathrm{D}^{\mu} \mathrm{LADRC}^{\mathrm{L}}$ controller design}

The ADRC method carries over the essence of the classical PID method and assimilates characteristics of the modern control theory. The traditional ADRC method consists of a tracking-differentiator (TD), a nonlinear state error feedback control law (NLSEF) and an extended state observer (ESO). The TD can coordinate the contradiction between rapidity and overshoot, the ESO can regard all disturbances as "unknown disturbances" $[25,26]$. Compared with the traditional ADRC method, the $\mathrm{FOPI}^{\lambda} \mathrm{D}^{\mu}$ ADRC method results in a $\mathrm{FOPI}^{\lambda} \mathrm{D}^{\mu}$ controller instead of the NLSEF.A new nonlinear FOPI $\lambda \mathrm{D} \mu$ ADRC method is proposed and adopted to hypersonic vehicle control problem, the structure diagram is shown in Fig. 3. The structure diagram of the hypersonic vehicle VM FOPI ${ }^{\lambda} D^{\mu}$ ADRC method is shown in Fig. 3.

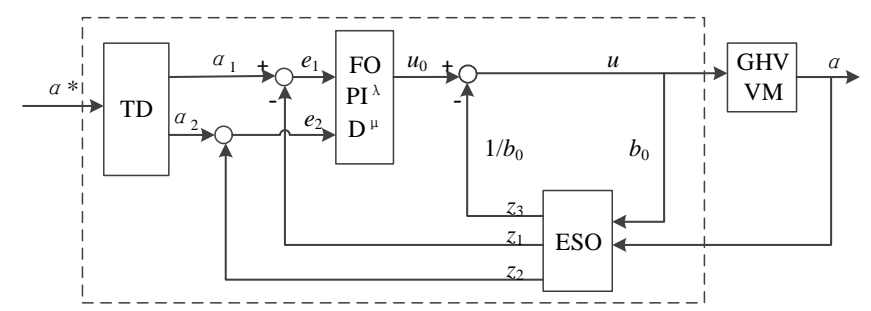

Fig. 3: The structure diagram of the VM FOPI ${ }^{\lambda}{ }^{\mu}$ ADRC method for hypersonic vehicle

In Fig. 3, the desired attack angle $\alpha^{*}$ is the input signal, the attack angle $\alpha$ is the output signal. TD, FOPI ${ }^{\lambda} \mathrm{D}^{\mu}$ and ESO inside dashed line frame are the proposed controllers. The controlled object GHV VM is the vertical model of a hypersonic vehicle. $\alpha_{1}$ and $\alpha_{2}$ are the tracking signal of $\alpha^{*}$ and derivative signal of $\alpha_{1}$ from the TD, respectively. $z_{1}, z_{2}$ and $z_{3}$ are the actual attack angle, the derivative signal of attack 
angle and unknown disturbances obtained from ESO, respectively. $u_{0}$ is the ideal control variable and $u$ is the actual control variable.

The TD discrete form can be described by the following equations:

$$
\begin{aligned}
& \alpha_{1}(k+1)=\alpha_{1}(k)+h \alpha_{2}(k) \\
& \alpha_{2}(k+1)=\alpha_{2}(k)+h\left(-r^{2}\left(\alpha_{1}(k)-\alpha^{*}(k)\right)-2 r \alpha_{2}(k)\right)
\end{aligned}
$$

where $\alpha_{1}(k)$ and $\alpha_{1}(k+1)$ denote estimated attack angle value of current time and next time, respectively, $\alpha_{2}(k)$ and $\alpha_{2}(k+1)$ are derivatives of $\alpha_{1}(k)$ and $\alpha_{1}(k+1)$, respectively, $r$ and $h$ represent speed factor and filtering factor, respectively. The larger $r$ values, the shorter the transition processes, the faster the response. The larger $h$ values, the better for the noise filtering.

The $\mathrm{FOPI}^{\lambda} \mathrm{D}^{\mu}$ equation is shown as follows:

$$
G_{c}(s)=\frac{U(s)}{E(s)}=K_{p}+\frac{K_{i}}{s^{\lambda}}+K_{d} s^{\mu}
$$

Where $\lambda$ and $\mu$ are restricted to $0<\lambda, \mu<1$. The $\mathrm{FOPI}^{\lambda} \mathrm{D}^{\mu}$ controller increases two degrees of freedom variables $\lambda$ and $\mu$, thus making the control affect more precisely and stable. The structure diagram of the $\mathrm{FOPI}^{\lambda} \mathrm{D}^{\mu}$ controller is shown in Fig. 4.

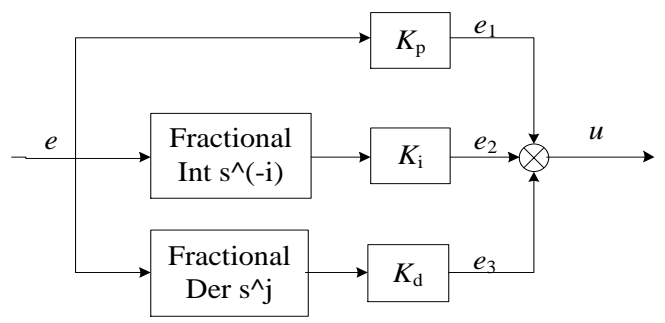

Fig. 4: The structure diagram of the FOPI $\lambda \mathrm{D} \mu$ controller

In Fig. 4, $e$ and $u$ represent the error and control variable, respectively, $e$ passes through $K_{p}$, FO integral method and FO derivative method to get $e_{1}, e_{2}$ and $e_{3}$, respectively.

The ESO in Fig. 3 is a third-order system and the extended state observer can be described by the following equation:

$$
\left\{\begin{array}{l}
e_{10}=z_{1}-\alpha \\
\dot{z}_{1}=z_{2}-\beta_{01} e_{10} \\
\dot{z}_{2}=z_{3}-\beta_{02}\left|e_{10}\right|^{\frac{1}{2}} \operatorname{sign}\left(e_{10}\right)+b_{0} u \\
\dot{z}_{3}=-\beta_{03}\left|e_{10}\right|^{\frac{1}{4}} \operatorname{sign}\left(e_{10}\right)
\end{array}\right.
$$

where $\beta_{01}, \beta_{02}$ and $\beta_{03}$ are adjustable parameters with different values, which can affect the effect of signal observed, $z_{1}$ and $z_{2}$ are the estimated attack angle $\alpha$ and estimated derivative signal of attack angle $\alpha$, respectively, $z_{3}$ is the estimated "unknown disturbances" of GHV VM and $b_{0}$ is to affect the compensation of unknown disturbances. With appropriate values of $\beta_{01}, \beta_{02}$ and $\beta_{03}$, the ESO can have good effect.

The LADRC method and the FOPI ${ }^{\lambda} \mathrm{D}^{\mu}$ ADRC method are almost the same, except for the ESO method. The ESO of the FOPI ${ }^{\lambda} \mathrm{D}^{\mu} \mathrm{ADRC}$ method is nonlinear, while the ESO of the LADRC method is linear (LESO), which can be shown as follows:

$$
\left\{\begin{array}{l}
e_{10}=z_{1}-\alpha \\
\dot{z}_{1}=z_{2}-\beta_{01} e_{10} \\
\dot{z}_{2}=z_{3}-\beta_{02} e_{10}+b_{0} u \\
\dot{z}_{3}=-\beta_{03} e_{10}
\end{array}\right.
$$

Where meanings of variables are the same as those in Equation 4.

Therefore, the structure diagram of a hypersonic vehicle VM LADRC method is shown in Fig. 5.

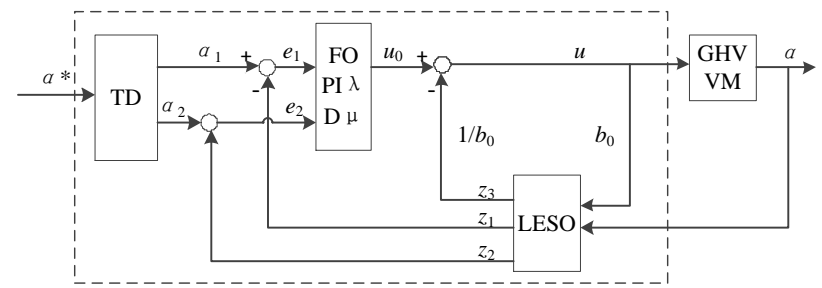

Fig. 5: The structure diagram of the hypersonic vehicle VM LADRC method

In Fig. 5, the LESO is different from the ESO in Fig. 3 and the other parts are the same, and the meanings of variables are the same as those in Fig. 3. The ADRC method and the LADRC method both have more tuning parameters than the traditional PID method, while the appropriate controller parameters depend on the experiences of experts. Sometime, the number of the ADRC method parameters can be reduced to one or two $[16,20]$. To compare the traditional ADRC method and the LADRC method clearly, this paper does not reduce parameters.

\subsection{Analysis of FOPI $\lambda \mathrm{D} \mu \mathrm{ADRC}$ method}

We have analyzed the stability of the second-order ESO and the FOPI ${ }^{\lambda} \mathrm{D}^{\mu} \mathrm{ADRC}$ method [28]. The stability analysis of LESO and the FOPI ${ }^{\lambda} D^{\mu}$ LADRC method is the same.

\subsubsection{The stability analysis of LESO}

The pitch channel equation (1) can be written as follows:

$$
\left\{\begin{array}{l}
\dot{\alpha}_{1}=\alpha_{2} \\
\dot{\alpha}_{2}=f(\cdot)+b_{0} u
\end{array}\right.
$$

Suppose the first-order derivative of $f(\cdot)$ exists and is bounded and define $\alpha_{3}=f(\cdot), f_{w}(t)=\dot{f}(\cdot)$, (6) can be extended to (7).The LESO for (6) is (8).

$$
\begin{gathered}
\left\{\begin{array}{l}
\dot{\alpha}_{1}=\alpha_{2} \\
\dot{\alpha}_{2}=\alpha_{3}+b_{0} u \\
\alpha_{3}=f(\cdot)
\end{array}\right. \\
\left\{\begin{array}{l}
e_{1}=z_{1}-\alpha_{1} \\
\dot{z}_{1}=z_{2}-\beta_{01} e_{1} \\
\dot{z}_{2}=z_{3}-\beta_{02} e_{1}+b_{0} u \\
\dot{z}_{3}=-\beta_{03} e_{1}
\end{array}\right.
\end{gathered}
$$

Define $e_{1}=z_{1}-\alpha_{1}, e_{2}=z_{2}-\alpha_{2}, e_{3}=z_{3}-\alpha_{3}$, from (7) and (8), the error equations can be shown as follows: 


$$
\begin{gathered}
\left\{\begin{array}{l}
\dot{e}_{1}=e_{2}-\beta_{01} e_{1} \\
\dot{e}_{2}=e_{3}-\beta_{02} e_{1} \\
\dot{e}_{3}=-\beta_{03} e_{1}-f_{w}(t)
\end{array}\right. \\
\left\{\begin{array}{l}
\dot{e}=A e+B u \\
A=\left(\begin{array}{lll}
-\beta_{01} & 1 & 0 \\
-\beta_{02} & 0 & 1 \\
-\beta_{03} & 0 & 0
\end{array}\right), B=\left(\begin{array}{l}
0 \\
0 \\
-1
\end{array}\right), u=f_{w}(t) \\
\left(\begin{array}{l}
E_{1}(s) \\
E_{2}(s) \\
E_{3}(s)
\end{array}\right)=(s I-A)^{-1} B F_{W}(s)=\left(\begin{array}{l}
\frac{s f(\cdot)}{s^{3}+\beta_{01} s^{2}+\beta_{00} s+\beta_{03}} \\
\frac{s\left(s+\beta_{01}\right) f(\cdot)}{s^{3}+\beta_{01} s^{2}+\beta_{02} s+\beta_{03}} \\
\frac{s\left(s^{2}+\beta_{01} s+\beta_{02}\right) f(\cdot)}{s^{3}+\beta_{01} s^{2}+\beta_{02} s+\beta_{03}}
\end{array}\right)
\end{array}\right.
\end{gathered}
$$

The characteristic equation of (9) is:

$$
s^{3}+\beta_{01} s^{2}+\beta_{02} s+\beta_{03}=0
$$

When $\beta_{01} \beta_{02}>\beta_{03}$, the LESO is stable. When $f(\cdot)$ is step function or ramp function, LESO is able to track $\alpha_{1}, \alpha_{2}, \alpha_{3}$. When $f(\cdot)$ is acceleration function, LESO is not able to track the desired signal.

\subsubsection{The stability analysis of $\mathrm{FOPI}^{\lambda} \mathrm{D}^{\mu} \mathrm{LADRC}$ method}

From Fig 5., $u_{0}$ and $u$ can be shown as

$$
\begin{aligned}
& \left\{\begin{aligned}
u_{0}= & \left(K_{p 1}+K_{i 1} s^{-\lambda}+K_{d 1} s^{u}\right)\left(\alpha^{*}-z_{1}\right) \\
& +\left(K_{p 2}+K_{i 2} s^{-\lambda}+K_{d 2} s^{u}\right)\left(\dot{\alpha}^{*}-z_{2}\right) \\
u= & u_{0}-\frac{z_{3}}{b_{0}}
\end{aligned}\right. \\
& \left\{\begin{aligned}
\dot{\alpha}_{1}= & \alpha_{2} \\
\dot{\alpha}_{2}= & \alpha_{3}-z_{3}+b_{0}\left(\left(K_{p 1}+K_{i 1} s^{-\lambda}+K_{d 1} s^{u}\right)\left(\alpha^{*}-z_{1}\right)\right. \\
& \left.+\left(K_{p 2}+K_{i 2} s^{-\lambda}+K_{d 2} s^{u}\right)\left(\dot{\alpha}^{*}-z_{21}\right)\right)
\end{aligned}\right.
\end{aligned}
$$

Let $\alpha_{1}=\alpha_{0}+\Delta \alpha, \alpha^{*}=\alpha_{0}+\Delta \alpha^{*}$,

$$
\begin{aligned}
\Delta \ddot{\alpha}= & -e_{3}+b_{0}\left(\left(K_{p 1}+K_{i 1} s^{-\lambda}+K_{d 1} s^{u}\right)\left(\Delta \alpha^{*}-\Delta \alpha-e_{1}\right)\right. \\
& \left.+\left(K_{p 2}+K_{i 2} s^{-\lambda}+K_{d 2} s^{u}\right)\left(\Delta \dot{\alpha}^{*}-\Delta \dot{\alpha}-e_{2}\right)\right)
\end{aligned}
$$

So the characteristic equation of (14) is:

$$
s^{2+\lambda}+b_{0} s\left(K_{p 2} s^{\lambda}+K_{i 2}+K_{d 2} s^{u+\lambda}\right)+b_{0}\left(K_{p 1} s^{\lambda}+K_{i 1}+K_{d 1} s^{u+\lambda}\right)=0
$$

Therefore, when parameters $K_{p 1}, K_{i 1}, K_{d 1}, K_{p 2}, K_{i 2}, K_{d 2}$ can make all roots of characteristic equation (11) are on the left half-plane, the FOPI $\lambda \mathrm{D} \mu \mathrm{LADRC}$ controller for hypersonic vehicles is stable.

\section{Comparative simulation of FOPI $\lambda D \mu$ ADRC and FOPI $^{\lambda} D^{\mu}$ LADRC}

Taking the longitudinal model of hypersonic vehicle as an example, the three modules of the auto disturbance rejection structure are tracking the differential device, the fractional order PID and the linear extended state observer. In order to compare and analyze the characteristics of the linear active disturbance rejection controller, the same structure of the active disturbance rejection controller is simulated, which is followed by the tracking controller, the fractional order PID and the extended state observer.

\subsection{Comparative simulation of normal operating conditions}

The angle of attack of the control system is a continuous square wave signal with amplitude of 10 degrees. The simulation results are shown in Fig. 6.

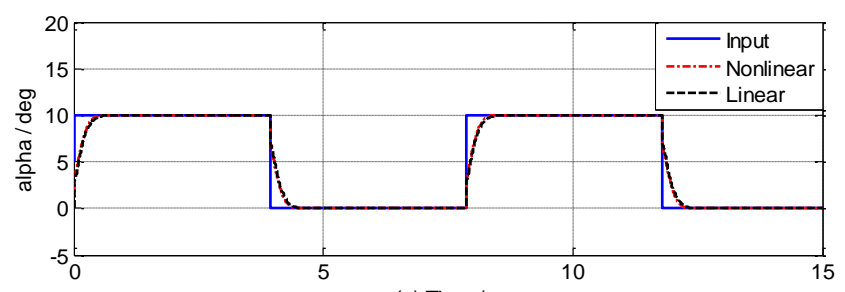

(a) Time / s

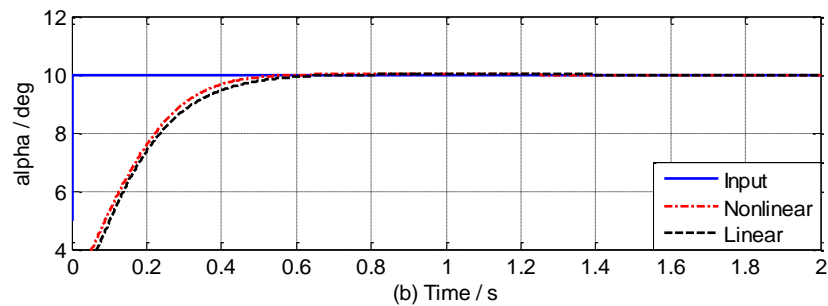


Fig. 6: Comparison of ADRC method and LADRC method without disturbances. (a) Comparison under disturbances; (b) Partial enlarged view of (a)

In Fig. 6, the 'Input' represents the continuous square wave signal; 'Nonlinear' is a nonlinear active disturbance rejection controller. 'Linear' is a linear active disturbance rejection controller. Two control structures can make the output of the attack angle of attack fast tracking input signal, with a very small steady-state error and overshoot. The adjustment time of linear structure and nonlinear structure is $0.322 \mathrm{~s}$ and $0.296 \mathrm{~s}$. Under the condition of no external disturbance, the control effect of the nonlinear active disturbance rejection control structure is better than that of the linear active disturbance rejection controller.

\subsection{Electronic Image Files (Optional)}

To show the anti-disturbance ability of the two controllers, the input signal is still a continuous wave signal with amplitude of 10 degrees. When the input signal is $2 \mathrm{~S}$ and $7 \mathrm{~S}$, the interference signal is added. The interference signal amplitude is 90 , and the duration is $140 \mathrm{~ms}$. The disturbance can be seen as an impact of the wind. The simulation results are shown in Fig. 7.
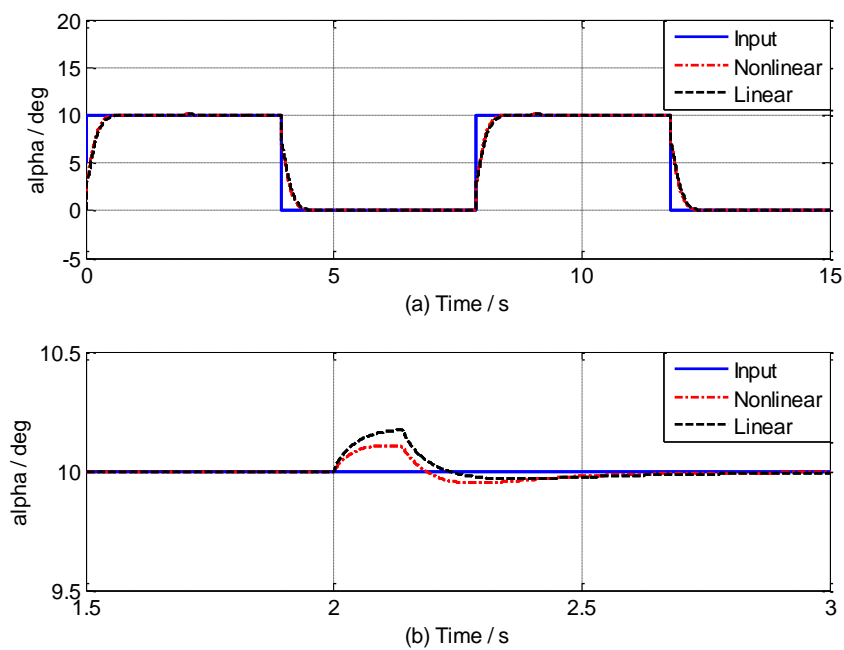

Fig. 7: Comparison of ADRC method and LADRC method under disturbances. (a) Comparison under disturbances; (b) Partial enlarged view of (a)

In Fig. 7, the meaning of each signal is the same as that of fig. 6.In Fig. 7(a), the two methods can track the reference signal rapidly. Two control structures are able to effectively track the input attack angle signal. In Fig. 7(b), the responses of ADRC method and LADRC method both have less than two percent changes lasting less than $1 \mathrm{~s}$ at $2 \mathrm{~s}$, when subjected to external disturbance. But, the response of ADRC method with respect to the disturbance is more stable. Therefore, for hypersonic vehicle vertical model, the nonlinear controller demonstrates nominal better anti-disturbance ability and stronger robustness than the linear controller in some degree.

\section{Conclusions}

In this paper, by combining $\mathrm{FOPI}^{\lambda} \mathrm{D}^{\mu}$ controller and the traditional ADRC method (TD, NLSEF and ESO), a $\mathrm{FOPI}^{\lambda} \mathrm{D}^{\mu}$ ADRC controller is designed for hypersonic vehicles. By replacing ESO with LESO, we obtain the FOPI ${ }^{\lambda} D^{\mu}$ LADRC controller. Then the differences of the FOPI ${ }^{\lambda} D^{\mu}$ ADRC method and FOPI $^{\lambda} D^{\mu}$ LADRC are firstly clarified and analyzed. The stability of LESO and $\mathrm{FOPI}^{\lambda} \mathrm{D}^{\mu}$ LADRC controller for hypersonic vehicle vertical model is analyzed. The experiment results show that the $\mathrm{FOPI}^{\lambda} \mathrm{D}^{\mu}$ ADRC method performs better than the FOPI ${ }^{\lambda} D^{\mu}$ LADRC method.

\section{References}

[1] D. Cheng, Controllability of switched bilinear systems, IEEE Trans. on Automatic Control, 50(4): 511-515, 2005.

[2] H. Poor, An Introduction to Signal Detection and Estimation. New York: Springer-Verlag, 1985, chapter 4.

[3] B. Smith, An approach to graphs of linear forms, accepted.

[4] D. Cheng, On logic-based intelligent systems, in Proceedings of 5 th International Conference on Control and Automation, 2005: 71-75.

[5] D. Cheng, R. Ortega, and E. Panteley, On port controlled hamiltonian systems, in Advanced Robust and Adaptive Control - Theory and Applications, D. Cheng, Y. Sun, T. Shen, and H. Ohmori, Eds. Beijing: Tsinghua University Press, 2005: 3-16.

[1] Gao G., Observer-Based Fault-Tolerant Control for an Air-Breathing Hypersonic Vehicle Model, Nonlinear Dynamics, 76(1): 409-430, 2014.

[2] Jiang B., Robust Fault-Tolerant Tracking Control for a Near-Space Vehicle Using a Sliding Mode Approach, System and Control Engineering, 225(8):1-12, 2011.

[3] Liu Z, Immersion and Invariance-Based Output Feedback Control of Air-Breathing Hypersonic Vehicles, IEEE Transactions on Automation Science \& Engineering, 2015:1-9.

[4] Wiese D.P., Adaptive Output Feedback Based on Closed-Loop Reference Models for Hypersonic Vehicles, Journal of Guidance, Control, and Dynamics, 2015: 1-12.

[5] Sun H. F., New Tracking-Control Strategy for Airbreathing Hypersonic Vehicles, Journal of Guidance, Control, and Dynamics, 36(3): 846-859, 2013.

[6] Cai G. H., Flight control system design for hypersonic reentry vehicle based on LFT-LPV method, Proc IMechE, Part G: $J$ Aerospace Engineering, 228(7):1130-1140, 2014.

[7] An H, Approximate Back-Stepping Fault-Tolerant Control of the Flexible Air-Breathing Hypersonic Vehicles, IEEE/ASME Transactions on Mechatronics, 2015:1-11.

[8] Li S. H., Composite Controller Design for an Air Breathing Hypersonic Vehicle, Proc IMechE, Part I: J Systems and Control Engineering, 226(5): 651-664, 2012.

[9] Duan H. B. and Li P., Progress in Control Approaches for Hypersonic Vehicle, Science China Technological Sciences, 55(10):2970-2965, 2012.

[10] Han J. Q., From the PID Technology to "Auto-Disturbance Rejection Control Technology, Control Engineering of China, 9(3):13-18, 2002.

[11] Guo B Z, Active disturbance rejection control approach to output feedback stabilization of a class of uncertain nonlinear systems subject to stochastic disturbance, IEEE Transactions on Automatic Control, 2015.

[12] Shi X. X., Motion Control of a Novel 6-degree-of-Freedom Parallel Platform Based on Modified Active Disturbance Rejection Controller, Proc IMechE, Part I: J Systems and Control Engineering, 228(2):87-96, 2014.

[13] Zhao L, Active Disturbance Rejection Position Control for a Magnetic Rodless Pneumatic Cylinder, IEEE Transactions on Industrial Electronics, 62(9):1-1, 2015.

[14] Shi X. X., Precision Motion Control of a Novel Electromagnetic Linear Actuator Based on a Modified Active 
Disturbance Rejection Controller, Proc IMechE, Part I: J Systems and Control Engineering, 226(5):606-614,2011.

[15] Sun G, Neural Active Disturbance Rejection Output Control of Multimotor Servomechanism, IEEE Transactions on Control Systems Technology, 23(2):746-753, 2015.

[16] Peng C, Mismatched Disturbance Rejection Control for Voltage-Controlled Active Magnetic Bearing via State-Space Disturbance Observer, IEEE Transactions on Power Electronics, 30(5):2753-2762, 2015.

[17] Tang Y. M., Linear Active Disturbance Rejection-Based Load Frequency Control Concerning High Penetration of Wind Energy, Energy Conversion and Management, 95: 259-271, 2015.

[18] Tan W, Linear Active Disturbance Rejection Control_ Analysis and Tuning via IMC, IEEE Transactions on Industrial Electronics, 2015:1-9.

[19] Ramírez-Neria M., Linear Active Disturbance Rejection Control of Underactuated Systems: the Case of the Furuta Pendulum, ISA Transactions, 53(4): 920-928, 2014.

[20] Sira-Ramírez H., Linear Observer-Based Active Disturbance Rejection Control of the Omnidirectional Mobile Robot, Asian Journal of Control, 15(1): 51-63, 2013.
[21] Zheng Q. L., Active Disturbance Rejection Control for Piezoelectric Beam, Asian Journal of Control. 16(6): 1612-1622, 2014

[22] Shaughnessy J. D., Hypersonic Vehicle Simulation Model: Winged-Cone Configuration, NASA Langley Research Center, 1990.

[23] Shahriar K., Development of An Aerodynamic Database for A Generic Hypersonic Air Vehicle, AIAA Guidance, Navigation, and Control Conference and Exhibit, 2005:2005-6257.

[24] Song J, Nonlinear Fractional Order Proportion-Integral-Derivative Active Disturbance Rejection Control Method Design for Hypersonic Vehicle Attitude Control, Acta Astronautica, 111:160-169, 2015.

[25] Han J. Q., From PID to Active Disturbance Rejection Control, IEEE Transactions on Industrial Electronics, 56(3): 900-906, 2009.

[26] Han J. Q., Active Disturbance Rejection Control Technologythe Technique for Estimating and Compensating the Uncertainties, Beijing: National Defense Industry Press, 2008:243-263. 